University at Albany, State University of New York

Scholars Archive

$9-2003$

\title{
Success, Truth, and the Galilean Strategy
}

P.D. Magnus

University at Albany, State University of New York, pmagnus@albany.edu

Follow this and additional works at: https://scholarsarchive.library.albany.edu/cas_philosophy_scholar

Part of the Philosophy Commons

\section{Recommended Citation}

Magnus, P.D., "Success, Truth, and the Galilean Strategy" (2003). Philosophy Faculty Scholarship. 45.

https://scholarsarchive.library.albany.edu/cas_philosophy_scholar/45

This Article is brought to you for free and open access by the Philosophy at Scholars Archive. It has been accepted for inclusion in Philosophy Faculty Scholarship by an authorized administrator of Scholars Archive. For more information, please contact scholarsarchive@albany.edu. 


\title{
Success, Truth, and the Galilean Strategy
}

\author{
P.D. Magnus \\ http://www.fecundity.com/job
}

Published in the British Journal for the Philosophy of Science, 54(3): 465-474. September 2003.

This is my final draft and so does not reflect copyediting done by the staff of the journal.

\begin{abstract}
Philip Kitcher develops the Galilean Strategy to defend realism against its many opponents. I explore the structure of the Galilean Strategy and consider it specifically as an instrument against constructive empiricism. Kitcher claims that the Galilean Strategy underwrites an inference from success to truth. We should resist that conclusion, I argue, but the Galilean Strategy should lead us by other routes to believe in many things about which the empiricist would rather remain agnostic.
\end{abstract}

1. Target: Empiricism

2. The Galilean Strategy

3. Strengthening the argument

4. Success and truth

5. Conclusion

\section{Target: Empiricism}

In Real Realism: The Galilean Strategy ([2001a], henceforth RR), Philip Kitcher offers a defense of realism from nearly half-a-dozen arguments against it. Against constructivists and empiricists, on matters both semantic and epistemic, he deploys the Galilean Strategy - a move to show that methods of settling questions about unobjectionable, observable matters should be relied on to settle questions about controversial, unobservable matters. (He develops the Galilean Strategy in less detail elsewhere ([2001b], ch. 2).) Here I will centrally concern myself with the response to van-Fraassen-style constructive empiricism, but my concerns may be raised with respect to the rest.

Kitcher outlines an argument from empiricist premises to the rejection of methods that putatively inform us about unobservables: We should only rely 
on methods that we can check independently. We can only check matters that we can observe. So, we should only rely on methods insofar as they inform us about observables. Therefore, we should remain agnostic about conclusions regarding unobservables. ${ }^{1}$

Kitcher notes that this argument has a long pedigree and sees Bas van Fraassen as its contemporary champion (RR, fn. 27 p. 166). In a recent paper, van Fraassen writes the following:

If you see a reflection of a tree in the water, you can also look at the tree and gather information about the geometric relations between the tree, the reflection, and your vantage point. The invariances in those relations are precisely what warrant the assertion that the reflection is a picture of the tree. If you say similarly about the microscopes images that they are pictures of e.g. paramecia, then you are asserting that there are certain invariant geometric relations between the object, image, and vantage point. But now you are postulating that these relations hold, rather than gathering information about whether that is so. ([2001], p. 160)

This is not yet an anti-realist conclusion. To show that we ought not believe in paramecia, one would need to show that we ought not postulate parameciasomething van Fraassen does not try to show. Rather than claiming that postulating paramecia is irrational, van Fraassen insists that it is not compelled by rationality. We may believe in paramecia or we may remain agnostic, as we choose. The former exposes us to the risk of being wrong when we might have avoided error, and the latter exposes us to the risk of not believing a truth that we might have believed. As William James observes, our obligations to gather truth and avoid error are continually in conflict. ${ }^{2}$ Van Fraassen only insists that, in light of this potential conflict, avoiding potential error by remaining agnostic about unobservables is permissible. Thus, Kitcher would need to do more than defeat the argument above in order to sway the constructive empiricist. He needs to provide a positive argument that empiricists should give up their agnosticism.

Indeed, Kitcher provides a positive argument. Examining the argumentative strategies that Galileo employed in convincing his contemporaries to believe what they could see in telescopes, he argues that they had good reason (as van Fraassen might put it) to postulate the moons of Jupiter, the rings of Saturn, and all the rest. Moreover, he claims that an analogue of Galileo's argument gives us good reason to accept other methods that inform us about unobservables.

\section{The Galilean Strategy}

In 1610, Galileo was faced with the problem of justifying the telescope as an instrument. One could see points of light when looking through the device toward Jupiter, but Galileo needed to show that the lights were moons and not not artefacts of the telescope itself. He did this 'by showing that the telescope 
would deliver conclusions that could be verified using methods that his contemporaries, including his critics, would accept' (RR, p. 173). He could take it out on a balcony, point it at a distant building, and anyone could see detail through it that they could only make out from a lesser distance with their naked eyes; this readily showed that the telescope was good for discerning details of structures in Northern Italy. After such tests, Kitcher notes, none of Galileo's interlocutors worried that the telescope would not work for such applications in the vicinity of London or Amsterdam. Within the terrestrial realm, there was no reason to draw a distinction between these places. As Kitcher writes: 'Galileo's central problem was to make the celestial-terrestrial distinction appear as irrelevant as the difference between London... and Venice' (RR, p. 174).

Kitcher analyzes Galileo's answer to this problem into two parts. First, Galileo exploited the vagueness of the boundary between the observable and the unobservable to show that the telescope was reliable beyond the bounds of what was straight-forwardly observable. Where only sharp-eyed observers could distinguish a fine detail unaided, both they and folks of ordinary acuity could make it out with the telescope. The deliverances of the telescope were thus shown to be continuous with the deliverances of plain vision - the unobservable was shown to be continuous with the observable. Importantly, this could be done for astronomical phenomena. Already, the boundary between the terrestrial and the celestial was softening. Second, Galileo argued directly against that boundary by cataloguing changes in the allegedly immutable heavens. Kitcher summarizes the action: 'Combining these two arguments with his ability to distribute telescopes that would generate an increasingly more consistent set of astronomical observations, Galileo was able to convince his peers that there was no more basis for thinking that the instrument was unreliable in the heavens than for believing it inept in some as yet untried part of the earth' (RR, p. 174).

Kitcher, not centrally concerned with the telescope, derives from Galileo's argument an argumentative schema that he dubs the Galilean Strategy. He generalizes in this way:

Methods of justification, like Galileo's telescope, can only be validated by examining the conclusions about observables to which they lead. It does not follow that the only conclusions licensed by those methods are conclusions about observables - any more than Galileo's demonstrations on buildings and ships only show that the telescope is reliable in Venice. We need to consider whether there are good reasons for distinguishing a method's usage in its application to observables from its usage in application to unobservables. (RR, p. $175)$

To distinguish it from the particular arguments made by Galileo, let's call the Galilean Strategy ' $\mathcal{G} S$ '. Take some method $M$ that provides the correct answers for matters we can check independently. $\mathcal{G} S$ may be summarized by the following schema:

$\mathcal{G S} 1 M$ provides correct answers up to and along the vague boundary between matters we can check independently of $M$ and ones that we cannot check. 
$\mathcal{G} S 2$ Prevailing reasons for thinking that the boundary might make a difference to the reliability of $M$ are mistaken.

$\therefore \quad M$ provides the correct answers for matters that we cannot check independently of $M$.

\section{Strengthening the argument}

There is an obvious way to employ $\mathcal{G} S$ against the constructive empiricist. To begin, Galileo's own arguments will do. Telescopes provide us a way of learning about the moons of Jupiter, the rings of Saturn, and many things more distant and exotic. ${ }^{3}$ A trivial variant of Galileo's argument might motivate belief in entities visible through optical microscopes: paramecia, cells, cellular organelles, and so on. We can use magnifying glasses and microscopes to clearly see things that we could otherwise see only with careful scrutiny, we can use them to see features which only our sharp-eyed friends can make out, and so on. The empiricist may resist these cases - distant things seen through the telescope could be made observable to everyone merely by bringing them closer, but this is not possible with the microscope. The empiricist may look to Hacking, who notes that the move 'from a magnifying glass to even a low powered microscope is the passage from what we might be able to observe with the eye unaided, to what we could not observe except with instruments' ([1985], p. 135). Nevertheless, there are intermediate cases for which we can confirm the things seen with the microscope. Hacking provides the example of microscopic metal grids used for reidentifying particular bodies on microscope slides. Grids of ordinary size are photographically reduced and metalized using techniques which operate also in the macroscopic realm ([1985], pp. 146-7). We can imagine making a series of grids, the largest clearly observable to the average person without any magnification and the smallest unobservable to even the keenest eyes. This series of cases would show that the microscope is reliable at and through the limits of what the average person can observe using only their unaided vision. ( $\mathcal{G} S 1$ is satisfied for the optical microscope.) There is no reason to believe that the operation of the microscope changes when we point it at things just beyond the acuity of our sharp-eyed friends. (GS2 is satisfied.) Thus, we draw the Galilean inference: Things we see in the microscope are really there.

Van Fraassen urges agnosticism about the deliverance of microscopes, but concedes, '. . I really don't mind very much if you reject this option for the optical microscope. I will be happy if you agree to it for the electron microscope. ... The point of constructive empiricism is not lost if the line is drawn in a somewhat different way from the way I draw it. The point would be lost only if no such line drawing is considered relevant to our understanding of science' ([2001], pp. 162-3). So a constructive empiricist can agree, in light of $\mathcal{G} S$, that there are paramecia and distant moons. Yet the genie of postulation, once let out of the bottle, is not so easily put back in.

Once we believe in the features that we can see with an optical microscope, 
we can employ $\mathcal{G} S$ again. The gross features that can be discerned with an electron microscope can be discerned with an optical microscope, and we can check them against each other up to the limits of optical magnification; see Hacking ([1985], p. 144). (GS1 is satisfied for the electron microscope.) There is no good reason to think that the electron microscope betrays us just beyond the limits of what we can check. (GS2 is satisfied.) So we should believe in things we can see with electron microscopes. Similar strategies can be used to extend the boundary of the observable whenever a new instrument has overlapping applications with one already vindicated by $\mathcal{G} S .{ }^{4}$

The constructive empiricist may reply that the boundary between the observable and the unobservable, vague though it may be, is principled. Take van Fraassen's preferred principle that observables are those entities and properties that are amenable to direct, unaided perception ([1980], p. 10). Paramecia are unobservable, but an application of $\mathcal{G} S$ directs us to believe in paramecia; so the constructive empiricist needs some way to deflect $\mathcal{G} S$. Note that the premise $\mathcal{G} S 2$ only asserts that prevailing arguments are insufficient to show that cases we cannot check would be different from cases that we can check. Why should the burden of proof lie that way? The constructive empiricist may insist that $\mathcal{G} S 2$ is insufficient and that there must be some positive reason to suppose that cases we cannot check would be like cases that we can check. If we accept this demand - and I can see no compelling reason not to- $\mathcal{G S}$ requires an extra premise:

$\mathcal{G} S 3$ There is some significant positive reason to think that the success of $M$ on matters we can check generalizes to matters that we cannot check.

Whereas $\mathcal{G} S 2$ obtains when we have no reason to think $M$ will fail beyond the limit of the observable, $\mathcal{G} S 3$ obtains only when we have some reason to think $M$ will succeed. ${ }^{5}$ To apply $\mathcal{G} S 3$, we need to show that there is continuity among the various applications of the method $M$. We might do this by marshalling systematic, theoretical resources. When $M$ is an instrument, though, we may begin with the homey observation that it is the same instrument used in the same way in both cases. A microscope is the same observable, material object when used to view the date on a penny and when used to look at a paramecium. Even when different lenses are used, the lenses may be made from the same glass and ground in the same way. The very material of the instrument provides continuity between cases in which it is used to look at observables and cases in which it is used to look at unobservables.

As Kitcher notes, Galileo's defense of the telescope involved relevant formulations of $\mathcal{G} S 1$ and $\mathcal{G} S 2$, but also an effort 'to distribute telescopes that would generate an increasingly more consistent set of astronomical observations' (RR, p. 174, cited above). Demonstrating the consistency of the instrument was a way of showing that telescopic observations of observables and unobservables were due to similar causes and thus that $\mathcal{G} S 3$ was satisfied. Whereas Kitcher offers this as a move in addition to $\mathcal{G} S$, it is plausibly seen as supporting a further premise of $\mathcal{G} S$. The fact that it was part of Galileo's strategy gives some reason to try and see it as part of the Galilean Strategy. 


\section{Success and truth}

Kitcher does not apply $\mathcal{G} S$ in the way I suggested in the previous section. Rather, he considers the realist inference from the success of a theory to the truth of that theory. A querist may entertain theories about matters which are temporarily unobservable to her; some theories will prove successful, others will not. Later, she can check for herself or confer with others to learn which theories were true and which false. She will find-Kitcher suggests - a strong, positive correlation between success and truth. Just as Galileo's interlocutors could view distant buildings through the telescope and later check the results, the querist notes which theories are successful and later checks to see that those are true. Taking inference from success to truth as $M, \mathcal{G} S 1$ is satisfied. It takes this form:

$\mathcal{G} S 1 \mathrm{k}$ Inferring truth from success provides correct answers up to and along the vague boundary between the observable and the unobservable.

Of course, 'success' must be understood in a rather strict way. If the querist's only goal is to give up smoking, then the correlation with truth will not be robust. 'Smoking is bad for me' might facilitate success, but so would 'Evil aliens will smite me if I light up again.' Kitcher constrains the type of success under consideration in several ways. First, he considers only success at prediction and at guiding intervention. Second, success must be over a large domain of applications that require fine-grained identification. Third, success must be at error-intolerant tasks. Finally, success should not be secured by compensatory errors. (RR, p. 179) Each restriction bars a way that the success-to-truth inference can fail. Consider a situation in which most any strategy would lead to a successful outcome, in which actual effort would be required in order to fail. In such situations, false theories might still support successful prediction and intervention. These cases are excluded by insisting that the task must be errorintolerant. The other conditions similarly exclude potential counter-examples to the correlation between success and truth. Supposing that all the major counter-examples have been excluded, $\mathcal{G} S 2$ is satisfied for the inference from success understood in this way to truth. ${ }^{6}$ We might rewrite it perspicuously in this way:

$\mathcal{G} S \mathbf{2 k}$ Whatever reasons we may have reasons for thinking the inference from success to truth would fail when applied to unobservables do not apply to the 'success' considered in $\mathcal{G} S 1 \mathrm{k}$.

Kitcher concludes from the instantiation of $\mathcal{G} S$ that we can infer the truth or approximate truth of scientific theories from their success.

Kitcher's application of $\mathcal{G S}$ has affinities with familiar realist arguments that reach this same conclusion. Realists claim that the best explanation of the success of science is the approximate truth of scientific theories. Yet anti-realists

reject inference to the best explanation. Realists reply that such abductive explanation is critical to science, even the bits of science that concern observables. 
Thus, one might argue that $\mathcal{G} S 1$ is satisfied for inference to the best explanation. Yet, the anti-realist replies, abductive inference in science is to the best causal explanation. Truth is not the cause of a theory's success. Peter Lipton explains: '. .. while scientific explanations are typically causal, the truth explanation is not. It is "logical": the truth of the theory entails the truth of its observed logical consequences, but it does not cause it' ([1994], p. 93). This gives us good reason to think that the inference to the best explanation that we can check is different from the inference to the best explanation that we cannot check, so $\mathcal{G} S 2$ is not satisfied. As such, $\mathcal{G} S$ will not underwrite inference to the best explanation.

Some realists claim that if the theories of science were not at least approximately true, then the success of science would be a miracle. Even if $\mathcal{G} S$ could be employed to support inferences from 'P would be a miracle' to 'not-P', the realist would have no comfort. Empiricists like van Fraassen insist that false theories could be successful even absent divine intervention.

Yet Kitcher does not employ $\mathcal{G} S$ to defend inference to the best explanation or the no-miracles argument. Rather, he defends the inference from success to truth directly. Unlike degenerate debates about abductive warrant, his argument does not rely on an intermediate principle of inference that empiricists already deny. The empiricist might deny the legitimacy of $\mathcal{G} S$, but it both has a straight-forward plausibility and is informed by venerable scientific practice. An obvious objection to $\mathcal{G} S$ can be answered by strengthening its assumptionsadding $\mathcal{G} S 3$. It takes this form:

$\mathcal{G} S 3 \mathbf{k}$ There is some significant positive reason to think that the reliability of success-to-truth inferences about observables generalizes to inferences about unobservables.

Supposing this three-premise version of $\mathcal{G} S$, the anti-realist can only eschew the success-to-truth rule by showing that one or more of the premises is not satisfied. It may be obvious that the empiricist's best target is $\mathcal{G} S 3$. When telescopes and microscopes are pointed at observables or unobservables, they are the same material instrument; as I argued above, this provides prima facie reason to think $\mathcal{G} S 3$ is satisfied. Yet in the case of successful theories, the theories are not instruments made of the same stuff as one another. They are not made of anything at all. Thus, the presumption of continuity of cases for the microscope cannot be extended to the success-to-truth inference. ${ }^{7}$ Why should we suppose that successful theories, even in a narrowly-defined sense, form a unified class of phenomena? Without some positive argument that what holds of successful theories of one sort will hold of other successful theories, the empiricist may reasonably refuse to generalize and thus reject the application of $\mathcal{G} S$.

The realist may resist the burden of proof represented by $\mathcal{G} S 3$. Just as the difference between the Earth and sky was as irrelevant to the operation of the telescope as the difference between Venice and London-Kitcher suggests - the difference between the observable and unobservable is irrelevant until proven 
relevant. Kitcher hopes to claim the high ground and set the presumption in favor of realism, but this begs the question against the empiricist. The VeniceLondon rhetoric does not show that $\mathcal{G} S 3$ is the wrong standard, since of course the difference between Italy and England was considered irrelevant to optics for positive reasons and not merely because no one could say why it should be relevant. ${ }^{8}$ Do we have comparable reasons to think that the success-veracity correlation will generalize? Kitcher says in summary:

In a nutshell, realists think that everyday experience supports a correlation between success and truth. They deny that empiricists can simply stipulate the limits of reliability of this correlation. Rather, those limits are to be charted in light of our best overall views about the ways in which the world works. (RR, p. 178)

Yet if $\mathcal{G} S 3$ is required for the application of $\mathcal{G} S$, the empiricist need not stipulate anything; reliability must be argued for rather than presumed, and it is the realist who must provide positive reasons for thinking the correlation will remain reliable. Kitcher has realist intuitions, but van Fraassen has empiricist intuitions. More will be needed than that. It will not do for the realist to say that whether the boundary of the observable is relevant or not is 'to be charted in light of overall views' about the world, because the empiricist and realist will cleave to different views.

\title{
5 Conclusion
}

Kitcher's reconstruction of the Galilean Strategy is informative. Careful application of $\mathcal{G S}$ may resolve outstanding questions in the philosophy of science; it is effective against the empiricist and perhaps against other anti-realists that come under Kitcher's sights. Nevertheless, it seems insufficient to support the realists' beloved connection between success and truth.

\section{Acknowledgements}

I first encountered the Galilean Strategy in seminars given by Philip Kitcher at the University of California, San Diego in 1997 and 1999. The present paper was prompted by discussion in the scientific realism reading group at UCSD in Winter 2002 and improved in light of comments from Craig Callender and Jay Odenbaugh.

\author{
P.D. Magnus \\ Department of Philosophy \\ Bowdoin College \\ 8400 College Station \\ Pols House, 5 Bath Road \\ Brunswick ME 04011-8484 \\ pmagnus@fecundity.com
}




\section{Notes}

${ }^{1}$ Kitcher calls this argument EEA (RR, pp. 161-2). He provides the argument in six steps, but nothing here turns on my truncating it as I have done.

${ }^{2}$ James ([1948], §VII). Longino similarly contrasts the 'knowledge-extending mission' of science with 'its critical mission' ([1990], p. 34).

${ }^{3}$ Van Fraassen allows that the moons of Jupiter are observable, since an astronaut in the vicinity of Jupiter would be able to see them without a telescope ([1980], p. 16). This is problematic, as Kitcher notes ([1993], p. 152-3). Should Galileo's contemporaries have objected by noting the then speculative nature of space travel?

${ }^{4}$ As Hacking notes, light microscopes and electron microscopes are both congeries of related instruments. Using $\mathcal{G} S$ to vindicate the all of them would require a great deal more than I can say here, but would proceed in a similar way by exploiting overlaps between the domains of a contested instrument and vindicated instruments.

${ }^{5}$ The problem of induction might be invoked as a reason to deny $\mathcal{G} S 3$ for any $M$, but both the realist and the constructive empiricist should resist such a move. The constructive empiricist must show that $\mathcal{G} S 3$ fails to obtain especially at the boundary between the observable and the unobservable, but the problem of induction plagues the unobserved as much as the unobservable.

${ }^{6}$ If there are further counter-examples, further monster-barring can ensure that $\mathcal{G} S 2$ is satisfied for some version of the realist inference.

${ }^{7}$ Insofar as theories are linguistic and instruments are causal devices, this parallels the objection that abductive inference to truth is not the same as abductive inference to causal explanation.

${ }^{8}$ Galileo and his contemporaries believed that the laws of physics would be invariant across space. More simply, travelling around doesn't seem to effect the behavior of light.

\section{References}

Hacking, I. [1985]: 'Do We See though a Microscope?', in P. M. Churchland and C. A. Hooker (eds.), 1985, Images of Science, Chicago: University of Chicago Press, pp. 132-52.

James, W. [1948]: 'The Will to Believe', in A. Castell (ed.), 1948, Essays in Pragmatism, New York: Hafner Publishing Co., pp. 88-109.

Kitcher, P. [1993]: The Advancement of Science, Oxford University Press. 
Kitcher, P. [2001a]: 'Real Realism: The Galilean Strategy', The Philosophical Review, v110 n2, pp. 151-97.

Kitcher, P. [2001b]: Science, Truth, and Democracy, Oxford University Press.

Lipton, P. [1994]: 'Truth, existence, and the best explanation', in A. A. Derksen (ed.), The Scientific Realism of Rom Harré, Tilburg University Press, pp. 89-111.

Longino, H. [1990]: Science as Social Knowledge, Princeton University Press. van Fraassen, B. C. [1980]: The Scientific Image, Oxford: Clarendon Press.

van Fraassen, B. C. [2001]: 'Constructive Empiricism Now', Philosophical Studies, 106, pp. 151-70. 